\title{
Collision Detection and Control of Parallel-structured Two-link Flexible Manipulators using Unscented Kalman Filter
}

\author{
Yuichi Sawada ${ }^{\dagger}$, Junki Kondo ${ }^{\ddagger}$ and Yusuke Watanabe ${ }^{\dagger}$ \\ Department of Mechanical and System Engineering, Kyoto Institute of Technology ${ }^{\dagger}$ \\ Matsugasaki Sakyo, Kyoto 606-8585, JAPAN \\ Department of Servo Motor Manufacturing, Mitsubishi Electric Corporation Nagoya Works ${ }^{\ddagger}$ \\ Yada-minami 5, Higashi, Nagoya 461-8670, JAPAN \\ Email: sawada@kit.ac.jp
}

\begin{abstract}
This paper presents a method of collision detection and control for parallel-structured two-link flexible manipulators based on the unscented Kalman filter. The exact dynamics of parallel-structured two-link flexible manipulators are described by quite complex nonlinear partial and ordinary differential equations. In this paper, the proposed parallel-structured two-link flexible manipulators are approximately modeled by a two-link flexible manipulator consisting of a couple of flexible beams with the same boundary conditions. In order to find the time when the flexible manipulator collides with the unlooked-for obstacle, the innovation process of the unscented Kalman filter which is one of the nonlinear state estimators is introduced. As the controller for the manipulator, the sliding mode controller is employed. In the normal situation, the sliding mode controller generates the control torques so that the tip position follows the reference trajectory. When the collision between the flexible manipulator and an unlooked-for obstacle is detected, the objective of the controller is switched from the position control to the suspend control, which is achieved by changing the reference trajectories. The performance of the proposed collision detection algorithm and controller is demonstrated by several numerical simulations.
\end{abstract}

\section{Introduction}

In recent years, flexible manipulators have received much attention. The feature of the flexible manipulators is the structure pursued the "lightweight" with the mechanical flexibility. Therefore, the flexible manipulators have advantages of lightweight and low energy consumption. Such flexible manipulators are useful for operations in various fields, e.g. space development pro-

\footnotetext{
${ }^{1}$ Part of this research is supported by the Japan Society for Promotion of Science (JSPS) under Grant-in-Aid for Scientific Research (C)-21560240.
}

grams, robotic assistants to humans and so forth. However, the derivation of their exact mathematical model and the accurate positioning control are exceptionally difficult because of undesirable vibrations due to low rigidity.

On the other hand, in use of the flexible manipulators in various environments, it is important to work out the safety operation of the manipulators, because there are varieties of obstacles (objects, human and so forth) in the work space. Therefore, it is necessary for the flexible manipulators to move with avoiding the obstacles. However, it is not always that the flexible manipulators could avoid unlooked-for obstacles in the work space. In order to reduce the damage, it is needed to introduce the active collision detection and suspend control algorithms to flexible manipulators. Unfortunately, there is no room to install the sensor which detects a collision in order to take advantage of the characteristic to be lightweight of flexible manipulators. The development of the collision detection mechanism based on the observation data for the flexible manipulators is significant and challengeable mission.

There are several researches on collision detection methods without extra sensors [1]-[4]. Moorehead and Wang proposed a collision detection method using strain gauges to determine the intensity and position of external force due to collision with a flexible cantilevered beam [4]. The estimation of the contact position in their approach was achieved by the mechanical relation between positions of the two strain gauges and the bending moments measured by the sensors. One of the authors proposed a method of collision detection for the single-link flexible manipulator using the innovation process of the Kalman filter [5],[6]. The authors presented a method of collision detection and suspend control for parallel-structured single-link flexible arms [7]. The collision is detected by using the innovation process of Kalman filter based on the measured data of strain sensors pasted on the side of the arm.

In this paper, we consider that our collision de- 
tection approach proposed in [7] is extended to the parallel-structured two-link flexible manipulator using unscented Kalman filter [8], which is one of the nonlinear filters. The feature of the parallel-structured flexible manipulator is that it holds the sufficient rigidity along the vertical axis and the mechanical flexibility along the displacement axis of the arm [9]. The exact mathematical model of parallel-structured two-link flexible manipulator is described by quite complex nonlinear partial and ordinary differential equations, because each link of the manipulator consists of a couple of flexible beams which are disposed parallel. In our research, each link of the parallel-structured two-link flexible manipulator is approximately modeled by a flexible beam with the same boundary conditions. The collision detection is realized using the innovation process of the state estimator.

In order to achieve collision detection and suspend control of the flexible manipulator, we employ the unscented Kalman filter (UKF) proposed by Julier et al. [10]-[12] and the sliding mode controller. The linearization of the exact mathematical model of parallelstructured two-link flexible manipulators is highly difficult because of its strong nonlinearity. However, the state estimator for parallel-structured two-link flexible manipulators can be constructed by using the UKF which is one of nonlinear state estimators. The intensity of the innovation process of the UKF abruptly becomes large when collision occurs. Introducing a scalar function based on the innovation process, the collision between the unlooked-for obstacles and the flexible manipulator can be detected if the scalar function exceeds the preassigned threshold.

As the controller, the control system for the nonlinear system model is required. For this purpose, the sliding mode controller is suitable for tracking and suspending control of manipulator. This controller has simple structure and robustness [13]. When the collision is detected, the objective of the controller is switched from the tracking control of the tip position to suspend control. The suspend control is achieved by replacing the reference trajectory by the tip position where the collision is detected.

\section{Mathematical Model}

\subsection{Parallel-structured Two-link Flexible Ma- nipulator}

Consider parallel-structured two-link flexible manipulators colliding with unknown obstacles illustrated in Fig.1. Each link of the manipulator consists of a couple of uniform Euler-Bernoulli beams. The end of each beam is clamped to a unit of hub and the other end is clamped to a tip-mass. Let $O_{1} X Y$ be the inertial Cartesian coordinate system; $O_{i} x_{i} y_{i}(i=1,2)$ the rotating coordinate systems rotated by the servomotor installed at $O_{1}$ and $O_{2} ; O_{i j} x_{i j} y_{j}(i, j=1,2)$ the rotating

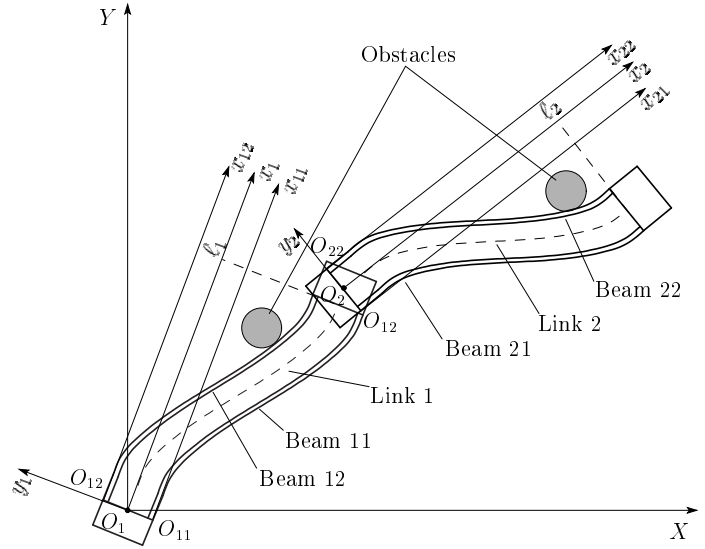

Fig. 1: Parallel-structured two-link flexible manipulator.

coordinate systems for Beam $i j$ of Link $i$, respectively.

The exact mathematical model of the parallelstructured two-link flexible manipulator is described by highly complex nonlinear differential equations. For the sake of simplicity, an approximated structure of parallel-structured two-link flexible manipulator is considered (See Fig.2). The principal components of mechanical displacements of the Beams $k 1$ and $k 2(k=$ $1,2)$ can be regarded as the same. Assuming that the effect of centrifugal force is sufficiently small, the mathematical models of both beams in each link become the same. For the sake of simplicity, we consider that each flexible link consisting of two beams can be mechanically approximated by one flexible beam with the same boundary conditions of the original them. Hence, the mathematical model of each flexible link can be described by a nonlinear partial differential equation.

\subsection{Simple-structured Two-link Flexible Ma- nipulator}

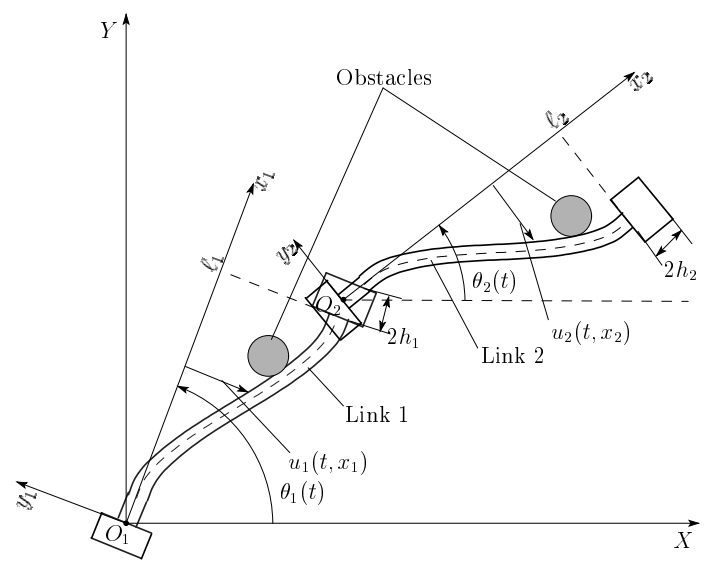

Fig. 2: Simple-structured model of parallel-structured two-link flexible manipulator.

Consider an simple-structured two-link flexible manipulator colliding with an unknown obstacle (Fig.2). 
It is assumed that the obstacle collides with either Link $1\left(x_{1}=x_{c 1}\right)$ or Link $2\left(x_{2}=x_{c 2}\right)$ at $t=t_{c}$, where $x_{c i}$ $(i=1,2)$ and $t_{c}$ are all unknown. $u_{i}\left(t, x_{i}\right)(i=1,2)$ denotes the transverse displacement of the Beam $i$ from $x_{i}$-axis. Let $\theta_{i}(t)$ be the angle between $O_{1} X$-axis and $O_{i} x_{i}$-axis. Physical parameters of the beams are as follows: $\rho$ the uniform mass density; $S$ the cross sectional area; $E I$ the uniform flexible rigidity (where $E$ denotes the Young's modulus and $I$ the second moment of cross sectional area); and $c_{D}$ the coefficient of Kelvin-Voigt type damping. The hub unit of the Link $i$ has the moment of inertia $J_{i}$. The tip-mass attached to the Link $i$ has the mass $m_{i}$ and the moment of inertia $J_{i+2}$. The mathematical model of the flexible manipulator expressed by the simplified structure can be derived by the Hamilton's principle. The derived mathematical model is given by the following Euler-Bernoulli type nonlinear partial differential equations and nonlinear ordinary differential equations:

$$
\begin{aligned}
& \rho S_{1} \ddot{u}_{1}\left(t, x_{1}\right)+c_{D} I_{1} \dot{u}_{1}^{\prime \prime \prime \prime}\left(t, x_{1}\right)+E I_{1} u_{1}^{\prime \prime \prime \prime}\left(t, x_{1}\right) \\
& \quad=-\rho S_{1} x_{1} \ddot{\theta}_{1}(t)+\rho S_{1} u_{1}\left(t, x_{1}\right) \dot{\theta}_{1}^{2}(t)+g_{1} \gamma_{1}\left(t, x_{1}\right) \\
& \quad+s_{1}(t) \delta\left(x_{1}-x_{c 1}\right)-R_{1}(\mathbf{p}) \delta\left(x_{1}-\ell_{1}\right) \\
& \rho S_{2} \ddot{u}_{2}\left(t, x_{2}\right)+c_{D} I_{2} \dot{u}_{2}^{\prime \prime \prime \prime}\left(t, x_{2}\right)+E I_{2} u_{2}^{\prime \prime \prime \prime}\left(t, x_{2}\right) \\
& \quad=-\rho S_{2}\left[\left\{L_{1} \cos \left\{\theta_{2}(t)-\theta_{1}(t)\right\}\right.\right. \\
& \left.\quad+u_{1}\left(t, \ell_{1}\right) \sin \left\{\theta_{2}(t)-\theta_{1}(t)\right\}\right\} \ddot{\theta}_{1}(t)+x_{2} \ddot{\theta}_{2}(t) \\
& \quad+\ddot{u}_{1}\left(t, \ell_{1}\right) \cos \left\{\theta_{2}(t)-\theta_{1}(t)\right\}+\left\{L_{1} \sin \left\{\theta_{2}(t)-\theta_{1}(t)\right\}\right. \\
& \left.\quad-u_{1}\left(t, \ell_{1}\right) \cos \left\{\theta_{2}(t)-\theta_{1}(t)\right\}\right\} \dot{\theta}_{1}^{2}(t) \\
& \quad-u_{2}\left(t, x_{2}\right)\left\{\dot{\theta}_{2}(t)-\dot{\theta}_{1}(t)\right\}^{2} \\
& \left.\quad+2 \dot{u}_{1}\left(t, \ell_{1}\right) \dot{\theta}_{1}(t) \sin \left\{\theta_{2}(t)-\theta_{1}(t)\right\}\right]+g_{2} \gamma_{2}\left(t, x_{2}\right) \\
& \quad+s_{2}(t) \delta\left(x_{2}-x_{c 2}\right)-R_{2}(\mathbf{p}) \delta\left(x_{2}-\ell_{2}\right) \\
& P_{11}(\mathbf{p}) \ddot{\theta}_{1}(t)+P_{12}(\mathbf{p}) \ddot{\theta}_{2}(t)+P_{13}(\mathbf{p}) \dot{\theta}_{1}(t)+P_{14}(\mathbf{p}) \dot{\theta}_{2}(t) \\
& +P_{15}(\mathbf{p}) \dot{\theta}_{1}^{2}(t)+P_{16}(\mathbf{p}) \dot{\theta}_{2}^{2}(t)-R_{s}(\mathbf{p})+Q_{11}(\mathbf{p}) \ddot{u}_{1}\left(t, \ell_{1}\right) \\
& +Q_{12}(\mathbf{p}) \dot{u}_{1}\left(t, \ell_{1}\right)=\tau_{1}(t)-\tau_{2}(t)+g_{3} \gamma_{\theta 1}(t) \\
& \quad+Q_{22}(\mathbf{p}) \ddot{u}_{2}\left(t, \ell_{2}\right)=\tau_{2}(t)+g_{4} \gamma_{\theta 2}(t), \\
& P_{21}(\mathbf{p}) \ddot{\theta}_{1}(t)+P_{22}(\mathbf{p}) \ddot{\theta}_{2}(t)+P_{23}(\mathbf{p}) \dot{\theta}_{1}(t)+P_{24}(\mathbf{p}) \dot{\theta}_{2}(t) \\
& \quad+P_{25}(\mathbf{p}) \dot{\theta}_{1}^{2}(t)+P_{26}(\mathbf{p}) \dot{\theta}_{2}^{2}(t)+Q_{21}(\mathbf{p}) \ddot{u}_{1}\left(t, \ell_{1}\right) \\
& \left.\quad+{ }_{1}\right)
\end{aligned}
$$

where $\mathbf{p}(t):=\left[\theta_{1}(t), \theta_{2}(t), u_{1}\left(t, x_{1}\right), u_{2}\left(t, x_{2}\right)\right]^{\mathrm{T}}$; the prime represents $\partial / \partial x_{i} ; L_{1}:=\ell_{1}+h_{1}, g_{i}(i=1, \cdots, 4)$ are constants; $\delta(\cdot)$ denotes Dirac's delta function and $\left.s_{i}(t)(i=1,2)\right)$ is the magnitude of collision input. $P_{i j}(\cdot)$ and $Q_{i j}(\cdot)$ are the nonlinear functions. $R_{1}(\cdot)$ represents the reaction force of the tip-mass and the Link $2 ; R_{2}(\cdot)$ the reaction force of tip-mass; and $R_{s}(\cdot)$ is the influence term of the collision. $s_{i}(t)(i=1,2)$ denotes the external force due to the collision. Assuming that the collision occurs momentarily, the magnitude of collision $s_{i}(t)(i=1,2)$ can be expressed by the following equation:

$$
s_{i}(t)=s_{i 0} \delta\left(t-t_{c}\right)
$$

where $s_{i 0}(i=1,2)$ is unknown parameter. The initial and boundary conditions of (1) and (2) are given $u_{i}\left(0, x_{i}\right)=\dot{u}_{i}\left(0, x_{i}\right)=0$ and $u_{i}(t, 0)=u_{i}^{\prime}(t, 0)=$ $u_{i}^{\prime}\left(t, \ell_{i}\right)=u_{i}^{\prime \prime \prime}\left(t, \ell_{i}\right)=0(i=1,2)$. The initial conditions of (3) and (4) are given by $\theta_{i}(0)=\theta_{0 i}$ and $\dot{\theta}_{i}(0)=0$.

The observation data is measured using the piezoelectric sensors and the potentiometers. The piezoelectric sensor with the length $b_{s}$ is pasted at the root of each link $\left(x_{i}=\xi_{i}\right)$, which measures the strain of the beams. The potentiometers are installed at the shaft of servomotors. The observations are given by the following manners:

$$
\begin{aligned}
& y_{i}(t)=a_{i} \int_{\xi_{i}}^{\xi_{i}+b_{s}} u_{i}^{\prime \prime}\left(t, x_{i}\right) d x_{i}+b_{i} \beta_{i}(t) \\
& y_{2+i}(t)=a_{2+i} \theta_{i}(t)+b_{2+i} \beta_{2+i}(t), \quad(i=1,2)
\end{aligned}
$$

where $a_{i}$ and $b_{i}$ are constants; and $\beta_{i}(t)$ represents the observation noise modeled by the white Gaussian noise.

\subsection{State Space Model}

Using the modal expansion method, the solutions of (1) and (2) can be represented by

$$
u_{i}\left(t, x_{i}\right) \cong \sum_{k=1}^{N} u_{i k}(t) \phi_{i k}\left(x_{i}\right), \quad(i=1,2)
$$

where $N$ denotes a sufficiently large positive number; $\phi_{i k}(\cdot)$ denotes the $k$-th eigenfunction (mode function) corresponding to the eigenvalue $\lambda_{i k}$ of the following eigenvalue problem:

$$
\frac{E I}{\rho S_{i}} \frac{d^{4}}{d x_{i}^{4}} \phi_{i k}\left(x_{i}\right)=\lambda_{i k} \phi_{i k}\left(x_{i}\right), \quad(i=1,2)
$$

with the boundary conditions

$$
\phi_{i k}(0)=\frac{d \phi_{i k}(0)}{d x_{i}}=\frac{d \phi_{i k}\left(\ell_{i}\right)}{d x_{i}}=\frac{d^{3} \phi_{i k}\left(\ell_{i}\right)}{d x_{i}^{3}}=0 .
$$

Introducing the state vector defined by

$$
\begin{gathered}
v(t)=\left[\dot{u}_{11}(t), \cdots, \dot{u}_{1 N}(t), \dot{u}_{21}(t), \cdots, \dot{u}_{2 N}(t),\right. \\
u_{11}(t), \cdots, u_{1 N}(t), u_{21}(t), \cdots, u_{2 N}(t), \\
\left.\dot{\theta}_{1}(t), \dot{\theta}_{2}(t), \theta_{1}(t), \theta_{2}(t)\right]^{\mathrm{T}},
\end{gathered}
$$

the state space model of the simple-structured two-link flexible manipulator and the observation model can be described by the following equations:

$$
\begin{aligned}
& \dot{v}(t)=A(v) v(t)+B(v) f(t) \\
&+G_{c}\left(v ; x_{c 1}, x_{c 2}\right) s(t)+G(v) \gamma(t) \\
& y(t)=C v(t)+E \eta(t),
\end{aligned}
$$

where $y(t)$ represents the observation vector defined by $y(t)=\left[y_{1}(t), \cdots, y_{4}(t)\right]^{\mathrm{T}} ; \quad A(v), \quad B(v)$ and $G(v)$ are matrices of nonlinear function of $v(t) ; \quad C$ and $E$ are constant coefficient matrices; 
$G_{c}\left(v ; x_{c 1}, x_{c 2}\right)$ is a nonlinear function of the state $v(t)$ and the collision positions $x_{c 1}$ and $x_{c 2} ; f(t):=$ $\left[\tau_{1}(t), \tau_{2}(t)\right]^{\mathrm{T}} ; \quad \gamma(t):=\left[\gamma_{11}(t), \cdots, \gamma_{1 N}(t), \gamma_{21}(t), \cdots\right.$, $\left.\gamma_{2 N}(t), \gamma_{\theta 1}(t), \gamma_{\theta 2}(t)\right]^{\mathrm{T}} ; \gamma_{i k}(t):=\int_{0}^{\ell_{i}} \gamma_{i}\left(t, x_{i}\right) \phi_{k}\left(x_{i}\right) d x_{i}$; $\eta(t):=\left[\beta_{1}(t), \cdots, \beta_{4}(t)\right]^{\mathrm{T}} ; \mathcal{E}\left\{\gamma(t) \gamma^{\mathrm{T}}(\tau)\right\}=W \delta(t-$ $\tau) ; \mathcal{E}\left\{\eta(t) \eta^{\mathrm{T}}(\tau)\right\}=V \delta(t-\tau)(\mathcal{E}\{\cdot\}$ : the mathematical expectation).

\section{Design of Unscented Kalman Filter}

The state space model described by (12) and (13) is a stochastic nonlinear system with collision input. In order to control the tip position and to reduce the random vibration of the whole flexible manipulator, the information of the state $v(t)$ is required. For these purposes, the unscented Kalman filter (UKF) for the following collision-free system is employed:

$$
\dot{v}_{f}(t)=A\left(v_{f}\right) v_{f}(t)+B\left(v_{f}\right) f(t)+G\left(v_{f}\right) \gamma(t),
$$

where $v_{f}(t)$ denotes the state vector of collision-free system.

The UKF is constructed for the discrete-time nonlinear stochastic system. However, the system (14) is the continuous-time system. In this paper, (14) is converted into the discretized version of (14). By using the Runge-Kutta method with the time interval $\Delta t,(13)$ and (14) are rewritten into

$$
\begin{aligned}
& v_{f}(k+1)=F\left(v_{f}(k), f(k), \gamma(k)\right) \\
& y(k)=C v(k)+E \eta(k),
\end{aligned}
$$

where $k$ denotes the time-step; and $F(\cdot)$ the nonlinear function calculated by (14)

The algorithm of UKF is summarized as the following three steps:

[Step 1]: The $(4 N+4)$-state vector $v_{f}(k)$ is approximated by $2(4 N+4)+1$ sigma points $\mathcal{X}_{i}$ with weight coefficients $W_{i}$ :

$$
\begin{aligned}
\mathcal{X}_{0} & =\hat{v}_{f}(k \mid k) \\
W_{0} & =\frac{\kappa}{n+\kappa} \\
\mathcal{X}_{i} & =\hat{v}_{f}(k \mid k)+\{\sqrt{(4 N+4+\kappa) P(k \mid k)}\}_{i} \\
W_{i} & =\frac{1}{2(n+\kappa)} \\
\mathcal{X}_{i+4 N+4} & =\hat{v}_{f}(k \mid k)-\{\sqrt{(4 N+4+\kappa) P(k \mid k)}\}_{i} \\
W_{i+4 N+4} & =\frac{1}{2(n+\kappa)}, \\
(i & =1, \cdots, 4 N+4)
\end{aligned}
$$

where $\kappa$ denotes the integer scaling parameter; $W_{i}$ the weight coefficient that is associated with the $i$-th point; and $\{\sqrt{(4 N+4+\kappa) P(k \mid k)}\}_{i}$ represents the $i$-th column of the matrix $U$ satisfying $M=U U^{\mathrm{T}}$ if $M:=$ $(4 N+4+\kappa) P(k \mid k)$. In this paper, the matrix $U$ is calculated via the incomplete Cholesky decomposition[14].
[Step 2]: Transform each point through the nonlinear function $F(\cdot)$, and the predicted mean, covariance and observation, the innovation covariance $P_{y y}(k+1 \mid k)$ and the cross correlation matrix $P_{v y}(k+1 \mid k)$ are computed as follows:

$$
\begin{aligned}
& \mathcal{X}_{i}(k+1 \mid k)=F\left(\mathcal{X}_{i}(k \mid k), f(k)\right) \\
& \hat{v}_{f}(k+1 \mid k)=\sum_{i=0}^{2(4 N+4)} W_{i} \mathcal{X}_{i}(k+1 \mid k) \\
& P(k+1 \mid k)=\sum_{i=0}^{2(4 N+4)} W_{i}\left\{\mathcal{X}_{i}(k+1 \mid k)-\hat{v}_{f}(k+1 \mid k)\right\} \\
& \times\left\{\mathcal{X}_{i}(k+1 \mid k)-\hat{v}_{f}(k+1 \mid k)\right\}^{\mathrm{T}}+G W G^{\mathrm{T}}(25) \\
& \mathcal{Y}_{i}(k+1 \mid k)=C \mathcal{X}_{i}(k+1 \mid k) 2(4 N+4) \\
& \hat{y}(k+1 \mid k)=\sum_{i=0} W_{i} \mathcal{Y}_{i}(k+1 \mid k) \\
& P_{y y}(k+1 \mid k)=\sum_{i=0}^{2(4 N+4)} W_{i}\left\{\mathcal{Y}_{i}(k+1 \mid k)-\hat{y}(k+1 \mid k)\right\} \\
& \times\left\{\mathcal{Y}_{i}(k+1 \mid k)-\hat{y}(k+1 \mid k)\right\}^{\mathrm{T}}+E V E^{\mathrm{T}} \\
& P_{v y}(k+1 \mid k)=\sum_{i=0}^{2(4 N+4)} W_{i}\left\{\mathcal{X}_{i}(k+1 \mid k)-\hat{v}_{f}(k+1 \mid k)\right\} \\
& \times\left\{\mathcal{Y}_{i}(k+1 \mid k)-\hat{y}(k+1 \mid k)\right\}^{\mathrm{T}} .
\end{aligned}
$$

[Step 3]: The state estimate and covariance are given through updating the prediction by the linear update rule which is specified by the weights chosen to minimize the mean squared error of the estimate. The update rule is

$$
\begin{aligned}
& \hat{v}_{f}(k+1 \mid k+1)=\hat{v}_{f}(k+1 \mid k) \\
& \quad+K(k+1)\{y(k+1)-\hat{y}(k+1 \mid k)\} \\
& P(k+1 \mid k+1)=P(k+1 \mid k) \\
& \quad-K(k+1) P_{y y}(k+1 \mid k) K^{\mathrm{T}}(k+1 \mid k),
\end{aligned}
$$

where $K(k+1)$ is the Kalman filter gain given by

$$
K(k+1)=P_{v y}(k+1 \mid k) P_{y y}^{-1}(k+1 \mid k) .
$$

\section{Collision Detection Algorithm}

It is an undesirable accident that the flexible manipulator collides with the unknown obstacle, because the collision input $s(t)$ affects the state of flexible manipulators as the disturbance. The problem of the collision detection is considered as a detection problem of the abrupt change from the collision-free system to the system with the collision. The change of the systems can be detected using the observation data measured by the piezoelectric sensors pasted at the root of all links. In 
other words, the collision is detected by making a decision whether the observation data $y(t)$ is provided from the collision included model or the collision-free model.

In order to detect the abrupt change of the system, the intensity of the innovation process is used. The innovation process of the UKF is defined by the difference between the actual observation and the estimate observation of collision-free system, i.e.,

$$
\mu(k)=y(k)-C \hat{v}_{f}(k \mid k),
$$

where $\hat{v}_{f}(k \mid k)$ is the estimate of $v_{f}(k)$ which is calculated by the UKF mentioned in the previous Section. Substituting (16) into (33), we have

$$
\mu(k)=C z(k)+E \eta(k),
$$

where $z(k)$ is the estimation error defined by $z(k):=$ $v(k)-\hat{v}_{f}(k \mid k)$. If the collision does not occurr, the state vector $v(k)$ is equal to $v_{f}(k)$. However, if the collision occurrs, $v(k)$ is equal to the state vector of collision model. In this case, $z(k)$ becomes large because of the collision input. In order to detect the collision, the following scalar function (collision detection function) is introduced:

$$
r(k)=\mu^{\mathrm{T}}(k) \mu(k) .
$$

If the collision detection function $r(k)$ exceeds a threshold $\varepsilon$, then the collision has occurred.

\section{Controller Design}

The mathematical model of the proposed flexible manipulator derived in Section 2 is the nonlinear stochastic system. The purpose of controller is to generate the control torques for the servomotors so that the tip position of the flexible manipulator follows the reference trajectory. For this purpose, the sliding mode controller is employed.

\subsection{Error System}

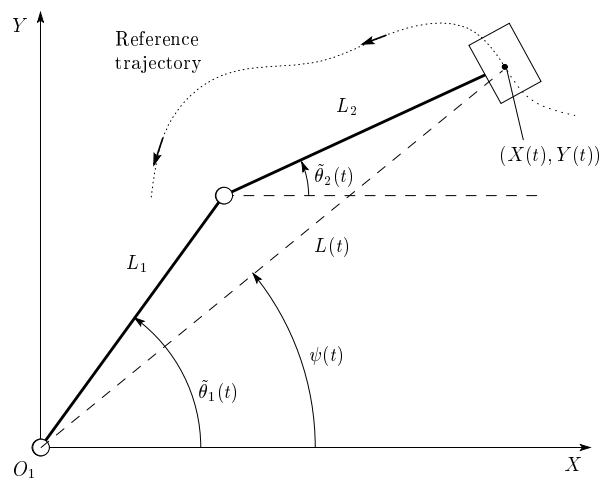

Fig. 3: The two-link rigid manipulator.

Consider that the tip position of the rigid manipulator is moved from the initial position to the desired position on the reference trajectory illustrated in Fig.3.
$(X(t), Y(t))$ denotes the tip position of the rigid manipulator. Let $\tilde{\theta}_{i}(t)(i=1,2)$ be the reference angle of the joint derived via the inverse kinematics:

$$
\begin{aligned}
& \tilde{\theta}_{1}(t)=\psi(t)+\cos ^{-1}\left\{\frac{L^{2}(t)+L_{1}^{2}-L_{2}^{2}}{2 L(t) L_{1}}\right\} \\
& \tilde{\theta}_{2}(t)=\tilde{\theta}_{1}(t)-\cos ^{-1}\left\{\frac{L^{2}(t)-L_{1}^{2}-L_{2}^{2}}{2 L_{1} L_{2}}\right\},
\end{aligned}
$$

where $L_{i}(i=1,2)$ represents the length of the rigid Link $i$; and $L(t)$ and $\psi(t)$ are given by

$$
\begin{aligned}
& L(t)=\sqrt{X^{2}(t)+Y^{2}(t)} \\
& \psi(t)=\tan ^{-1}\left\{\frac{Y(t)}{X(t)}\right\} .
\end{aligned}
$$

The flexible manipulator is controlled so that its state converges to the equilibrium sate which is given by the state of rigid manipulator, i.e. $v_{e q}(t):=\left[O_{1 \times 4 N}\right.$ $\left., \dot{\tilde{\theta}}_{1}, \dot{\tilde{\theta}}_{2}, \tilde{\theta}_{1}, \tilde{\theta}_{2}\right]$, where $O_{1 \times 4 N}$ expresses the $1 \times 4 N$ zero vector. The error state vector $v_{e}(t)$ is defined by

$$
\begin{gathered}
v_{e}(t):=v(t)-v_{e q}(t) \\
\equiv\left[\dot{u}_{11}(t), \cdots, \dot{u}_{1 N}(t), \dot{u}_{21}(t), \cdots, \dot{u}_{2 N}(t),\right. \\
u_{11}(t), \cdots, u_{1 N}(t), u_{21}(t), \cdots, u_{2 N}(t), \\
\left.\dot{e}_{1}(t), \dot{e}_{2}(t), e_{1}(t), e_{2}(t)\right]^{\mathrm{T}},
\end{gathered}
$$

where $e_{i}(t)(i=1,2)$ denotes $e_{i}(t):=\theta_{i}(t)-\tilde{\theta}_{i}(t)$. Introducing $v_{e}(t)$, the error system corresponding to (12) can be described by

$$
\dot{v}_{e}(t)=A\left(v_{e}(t)\right) v_{e}(t)+B\left(v_{e}(t)\right) f(t) .
$$

If the flexible manipulator is well controlled, the amplitude of $v_{e}(t)$ can be regarded sufficiently small, i.e. $\left\|v_{e}(t)\right\| \ll 1$. In this paper, we assume that $A\left(v_{e}(t)\right) \cong$ $A(0)$ and $B\left(v_{e}(t)\right) \cong B(0)$. Using these approximations, the error system is rewritten into the following expression:

$$
\dot{v}_{e}(t)=A_{e} v_{e}(t)+B_{e} f(t),
$$

where $A_{e}$ and $B_{e}$ are constant matrices denoted by

$$
A_{e}:=A(0), \quad B_{e}:=B(0) .
$$

\subsection{Sliding Mode Controller}

The objective of the sliding mode controller is to track the reference trajectory by the tip and to suppress of the random vibration of the manipulator. Another objective of this controller is to suspend the motion of the manipulator when the collision is detected.

The control input is given by

$$
f(t)=-\left(S B_{e}\right)^{-1} S A_{e} v_{e}(t)-F \frac{\sigma(t)}{\|\sigma(t)\|+\delta} .
$$

where $S$ is the hyperplane; $\delta$ is a positive constant; $F$ is the nonlinear controller gain; and $\sigma(t)$ is the switching 
function. There are several methods to determine the matrix $S$. In this research, a method to choose $S$ as a feedback gain of the optimal control is used. Namely, $S$ is determined as follows[13]:

$$
\begin{aligned}
& S=B_{e}^{\mathrm{T}} P \\
& P A_{e}+A_{e}^{\mathrm{T}} P-P B_{e} B_{e}^{\mathrm{T}} P+Q=0,
\end{aligned}
$$

where $Q$ denotes non-negative symmetric matrix which is the weight matrix used in the following cost functional:

$$
\begin{aligned}
J= & \int_{0}^{T}\left\{\int _ { 0 } ^ { \ell _ { 1 } } \left[q_{1}\left\{\dot{u}_{1}\left(t, x_{1}\right)\right\}^{2}+q_{2}\left\{u_{1}\left(t, x_{1}\right)\right\}^{2}\right.\right. \\
& \left.+q_{3}\left\{\dot{u}_{1}^{\prime \prime}\left(t, x_{1}\right)\right\}^{2}+q_{4}\left\{u_{1}^{\prime \prime}\left(t, x_{1}\right)\right\}^{2}\right] d x_{1} \\
& +\int_{0}^{\ell_{2}}\left[q_{5}\left\{\dot{u}_{2}\left(t, x_{2}\right)\right\}^{2}+q_{6}\left\{u_{2}\left(t, x_{2}\right)\right\}^{2}\right. \\
& \left.+q_{7}\left\{\dot{u}_{2}^{\prime \prime}\left(t, x_{2}\right)\right\}^{2}+q_{8}\left\{u_{2}^{\prime \prime}\left(t, x_{2}\right)\right\}^{2}\right] d x_{2} \\
& \left.+q_{9} \dot{e}_{1}^{2}(t)+q_{10} e_{1}^{2}(t)+q_{11} \dot{e}_{2}^{2}(t)+q_{12} e_{2}^{2}(t)\right\} d t \\
\equiv & \int_{0}^{T} v_{e}^{\mathrm{T}}(t) Q v_{e}(t) d t,
\end{aligned}
$$

where $Q:=\operatorname{diag}\left\{\Theta_{1}, \Theta_{3}, \Theta_{2}, \Theta_{4}, q_{9}, q_{11}, q_{10}, q_{12}\right\} ; \Theta_{1}=$ $q_{1} I_{N}+q_{3} \Psi_{1} ; \Theta_{2}=q_{2} I_{N}+q_{4} \Psi_{1} ; \Theta_{3}=q_{5} I_{N}+q_{7} \Psi_{2} ;$ $\Theta_{4}=q_{6} I_{N}+q_{8} \Psi_{2}\left(I_{N}: N\right.$-dimensional unit matrix $)$; and

$\Psi_{i}=\left[\begin{array}{ccc}\int_{0}^{\ell_{i}} \phi_{i 1}^{\prime \prime}\left(x_{i}\right) \phi_{i 1}^{\prime \prime}\left(x_{i}\right) d x_{i}, & \cdots & , \int_{0}^{\ell_{i}} \phi_{i 1}^{\prime \prime}\left(x_{i}\right) \phi_{i N}^{\prime \prime}\left(x_{i}\right) d x_{i} \\ \vdots & \vdots \\ \int_{0}^{\ell_{i}} \phi_{i N}^{\prime \prime}\left(x_{i}\right) \phi_{i 1}^{\prime \prime}\left(x_{i}\right) d x_{i}, & \cdots & , \int_{0}^{\ell_{i}} \phi_{i N}^{\prime \prime}\left(x_{i}\right) \phi_{i N}^{\prime \prime}\left(x_{i}\right) d x_{i}\end{array}\right]$.

The switching function is defined by

$$
\sigma(t)=S v_{e}(t)
$$

and nonlinear controller gain must satisfy the following condition:

$$
F\left\{\begin{array}{l}
>0: \text { if } S B_{e}>0 \\
<0: \text { if } S B_{e}<0 .
\end{array}\right.
$$

Because of using the unscented Kalman filter, it is necessary that the switching function $\sigma(t)$ and the controller input $f(t)$ given by the sliding mode controller are converted into the discretized version as the following equations:

$$
\begin{aligned}
\sigma(k) & =S v_{e}(k) \\
f(k) & =-\left(S B_{e}\right)^{-1} S A_{e} v_{e}(k)-F \frac{\sigma(k)}{\|\sigma(k)\|+\delta} .
\end{aligned}
$$

\subsection{Suspend Control}

When the collision is detected, it is necessary that the flexible manipulator is suspended for absorbing the impact force of collision. The proposed flexible manipulator is controlled so that the tip position tracks the reference trajectory using the sliding mode controller. In order to suspend the motion of the manipulator, we consider that the reference trajectory is switched to another trajectory when the collision occurs. The tip position of flexible manipulator at the time $t_{c}$ when the collision occurs is defined by

$$
\left(X\left(t_{c}\right), Y\left(t_{c}\right)\right)=:\left(X_{c}, Y_{c}\right),
$$

where $X_{c}$ and $Y_{c}$ are constants. The idea of the suspend control is achieved by fixing the reference trajectory at $\left(X_{c}, Y_{c}\right)$ after the collision is detected, i.e.

$$
\left\{\begin{array}{l}
X(t)=X_{c} \\
Y(t)=Y_{c} .
\end{array} \quad\left(t>t_{c}\right)\right.
$$

\section{Simulation Results}

In this section, the several numerical results are presented. The flexible beams are assumed to be made with the phosphor bronze. The physical parameters and the coefficients of the flexible manipulator are listed in Table 1. The observation data is supposed to be measured by the piezoelectric sensors with their length $b_{s}=3 \times 10^{-2}[\mathrm{~m}]$ and width $1.2 \times 10^{-2}[\mathrm{~m}]$ pasted at $\xi_{i}=3 \times 10^{-3}[\mathrm{~m}](i=1,2)$ and the potentiometers are installed at respective hubs. The parameters of the observation system were set as $a_{i}=b_{i}=1.0(i=$ $1, \cdots, 4)$. The covariance matrices for the system and observation noises were given by $W=10^{-5} \times I_{4 N+4}$, $V=10^{-8} \times I_{4}$. The number of modes of the flexible arms was set as $N=2$. The time partition in this numerical simulations was set as $\Delta t=1 \times 10^{-3}[\mathrm{~s}]$.

Table 1: Physical parameters of flexible manipulators.

\begin{tabular}{cl}
\hline Parameters & Values \\
\hline$\ell_{1}$ & $0.3[\mathrm{~m}]$ \\
$\ell_{2}$ & $0.28[\mathrm{~m}]$ \\
$\mathrm{E}$ & $1.1 \times 10^{5}[\mathrm{MPa}]$ \\
$S_{1}$ & $4 \times 10^{-5}\left[\mathrm{~m}^{2}\right]$ \\
$S_{2}$ & $8 \times 10^{-6}\left[\mathrm{~m}^{2}\right]$ \\
$\rho$ & $8.8 \times 10^{3}\left[\mathrm{~kg} / \mathrm{m}^{2}\right]$ \\
$c_{D}$ & $4.84 \times 10^{4}\left[\mathrm{~N} \cdot \mathrm{s} / \mathrm{m}^{2}\right]$ \\
$J_{1}$ & $0.01\left[\mathrm{~kg} \cdot \mathrm{m}^{2}\right]$ \\
$J_{2}$ & $0.035\left[\mathrm{~kg} \cdot \mathrm{m}^{2}\right]$ \\
$J_{3}$ & $0.035\left[\mathrm{~kg} \cdot \mathrm{m}^{2}\right]$ \\
$J_{4}$ & $0.002\left[\mathrm{~kg} \cdot \mathrm{m}^{2}\right]$ \\
$m_{1}$ & $0.3[\mathrm{~kg}]$ \\
$m_{2}$ & $0.02[\mathrm{~kg}]$ \\
$h_{1}$ & $0.04[\mathrm{~m}]$ \\
$h_{2}$ & $0.01[\mathrm{~m}]$ \\
$g_{i}(i=1, \cdots, 4)$ & 1.0 \\
\hline
\end{tabular}




\subsection{Tracking Control}

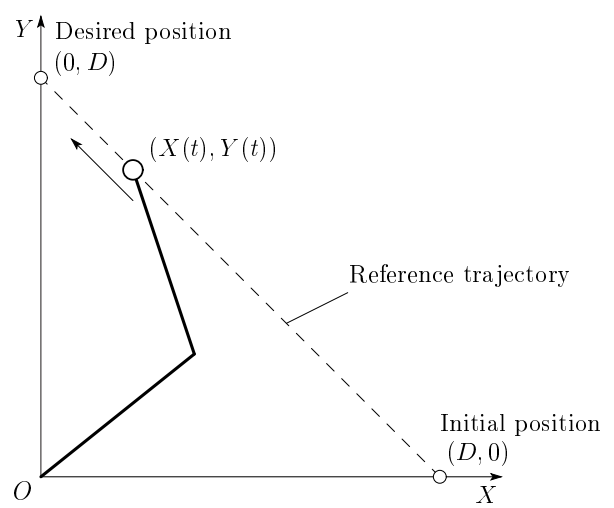

Fig. 4: Reference trajectory given by straight line for the tip of flexible manipulator.

Consider the straight line as the reference trajectory illustrated in Fig.4. Let the position of the tip be $(X(t), Y(t))$. The straight line is defined by

$$
X(t)+Y(t)=D,
$$

where $D\left(\leq L_{1}+L_{2}\right)$ denotes the positive constant. Then, the tip of the flexible manipulator moves to the desired position $(0, D)$ from the initial position $(D, 0)$. In this simulation, let $D=0.5[\mathrm{~m}]$, and the tip of the flexible manipulator assumed to reach the desired position at $t=2[\mathrm{~s}]$. The initial conditions were set as $u_{i j}(0)=0[\mathrm{~m}], \dot{u}_{i j}(0)=0[\mathrm{~m} / \mathrm{s}], \dot{\theta}_{i}(0)=0[\mathrm{rad} / \mathrm{s}]$, $\theta_{1}(0)=0.60[\mathrm{rad}]$ and $\theta_{2}(0)=-0.72[\mathrm{rad}]$. The initial condition of the state vector of the control error system was set as zero. The reference angles are calculated by $(36) \sim(39)$. The weight coefficients in the cost functional were set as $q_{1}=50, q_{2}=1000, q_{3}=500, q_{4}=10$, $q_{5}=500, q_{6}=1000, q_{7}=500, q_{8}=10, q_{9}=10$, $q_{10}=5000, q_{11}=10, q_{12}=5000$. The nonlinear controller gain was $F=1, \delta=0.5$. The simulation study was carried out for 3 seconds. The resultant path of the tip mass in the case of the tracking control shown in Fig. 5 was computed by

$$
\begin{aligned}
X(t)= & L_{1} \cos \theta_{1}(t)-u_{1}\left(t, \ell_{1}\right) \sin \theta_{1}(t)+L_{2} \cos \theta_{2}(t) \\
& -u_{2}\left(t, \ell_{2}\right) \sin \theta_{2}(t) \\
Y(t)= & L_{1} \sin \theta_{1}(t)+u_{1}\left(t, \ell_{1}\right) \cos \theta_{1}(t)+L_{2} \sin \theta_{2}(t) \\
& +u_{2}\left(t, \ell_{2}\right) \cos \theta_{2}(t),
\end{aligned}
$$

where

$$
u_{i}\left(t, \ell_{i}\right)=\sum_{k=1}^{2} u_{i k}(t) \phi_{i k}\left(\ell_{i}\right) .
$$

As seen in Fig.5, the tip position of the flexible manipulator have nearly traced the target trajectory.

\subsection{Collision Detection and Suspend Control}

The numerical simulation of collision detection and suspend control of two-link flexible manipulator is carried out. The suspend trajectory in this case is the

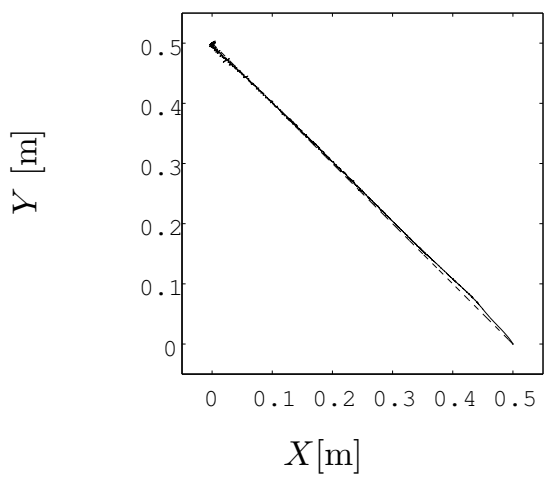

Fig. 5: Behavior of tip position of the flexible manipulator controlled by the sliding mode controller. In this figure, the dashed line depicts the reference trajectory and the solid line represents the tip's trajectory of the flexible manipulator calculated by (56) and (57).

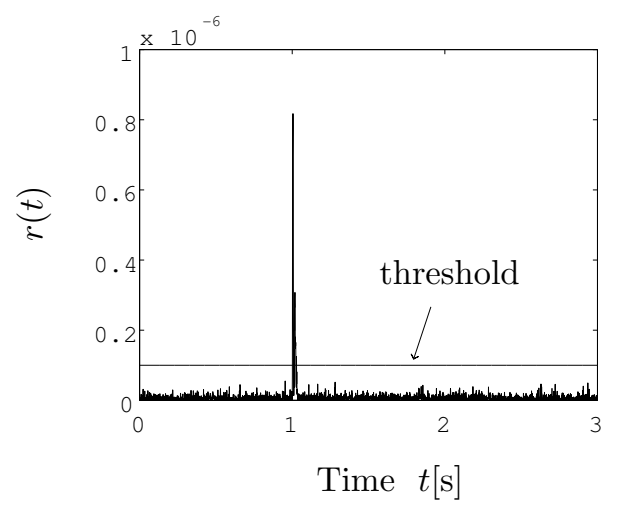

Fig. 6: Behavior of the collision detection function $r(t)$ in the case that an obstacle collides with the Link 1 $\left(x_{c 1}=0.15[\mathrm{~m}], t_{c}=1[\mathrm{~s}], \theta_{2}(t)=0[\mathrm{rad}]\right)$.

same used in the previous subsection (See Fig.4). After the collision is detected, the fixed position is used as the reference trajectory which is given by (54). The collision is assumed to occur at $t_{c}=1[\mathrm{~s}]$. The threshold is set as $\varepsilon=1 \times 10^{-7}$.

The numerical results in the case that the obstacle collides with Link 1 are considered. Fig.6 shows the time history of the collision detection function $r(t)(t=k \Delta t)$. It seems that $r(t)$ has abruptly increased at $t=1[\mathrm{~s}]$. The value of the collision detection function has exceeded the threshold $\varepsilon$ at the time when the collision occurred. Fig.7 shows the trajectory of the tip position in this case. The tip position changed greatly due to the collision input when the collision was detected, but suspended at the position that the collision had occurred.

\section{Conclusions}

This paper has presented a method of collision detection and control for parallel-structured two-link flexi- 


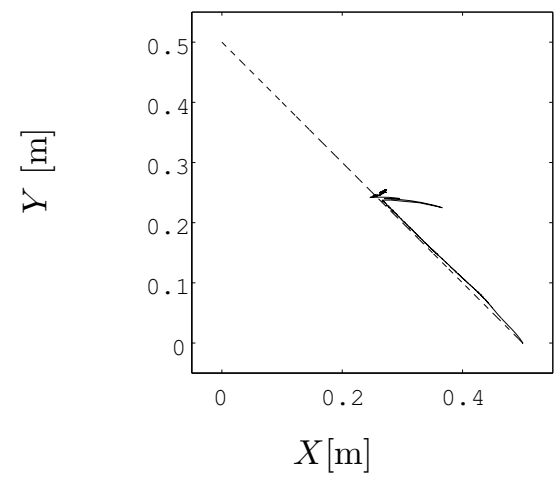

Fig. 7: Trajectory of the tip position of flexible manipulator when the collision is detected at Link 1 at $t_{c}=1[\mathrm{~s}]$. The dashed line depicts the reference trajectory and the solid line represents the resultant path of the flexible manipulator calculated by (56) and (57).

ble manipulators. The exact mathematical model of parallel-structured two-link flexible manipulators was described as a nonlinear distributed parameter system. In this paper, the mathematical model of the manipulator was converted into finite-dimensional system by using the modal expansion technique. Then, the UKF and the sliding mode controller were designed for the finite-dimensional system. The collision detection was achieved by using the innovation process of the UKF, because the two-link flexible manipulator was one of the nonlinear systems. The unexpected input caused by collisions of the unlooked-for obstacles excites an undesirable vibration of the flexible manipulator. Introduced collision detection function $r(t)$ has been successfully detected when the unlooked-for obstacles have collided with the manipulator.

The controller for the flexible manipulator was designed via the sliding mode control theory. The objectives of the controller were to reduce the random vibration of the flexible manipulator depending on the mechanical flexibility and to control the position of the tip-mass so that the tip of the flexible manipulator follows the reference trajectory. The suspend control was achieved by switching the reference trajectories from the normal control phase to the suspend control phase when the collision was detected.

By performing the numerical simulations, the efficiency of the collision detection function and the sliding mode controller based on the unscented Kalman filter was confirmed. The control of the tip of the flexible manipulator has been worked out.

\section{References}

[1] T. Matsumoto and K. Kosuge: Collision detection of manipulator based on adaptive control law, Proc. 2001 IEEE/ASME Int. Conf. on Advanced Intelligent Mechanics, 177-182, 2001.
[2] A. Garcia, V. Feliu and J. A. Somolinos: Experimental testing of a gauge based collision detection mechanism for a new three-degree-of-freedom flexible robot, J. Robotics Systems, 20-6, 271-284, 2003.

[3] M. Kaneko, N. Kanayama and T. Tsuji: Active antenna for contact sensing, IEEE Trans. on Robotics and Automation, AC-14-2, 278-291, 1998.

[4] S. Moorehead and D. Wang: Collision detection using a flexible manipulator: a feasibility study, Proc. IEEE Int. Conf. on Robotics and Automation, 804-809, 1996.

[5] Y. Sawada: On-line Collision Detection for Flexible Cantilevered Beams Using Innovation Process, Trans. of ISCIE, 17-8, 349-357 (in Japanese), 2004.

[6] Y. Sawada: Collision Detection and Control of a Single-link Flexible Arm, Trans. of ISCIE, 19-6, 250-261 (in Japanese), 2006.

[7] J. Kondo and Y. Sawada: Collision Detection and Suspend Control of Parallel-structured Single-link Flexible Arms, Proc. SICE Annual Conference. 2008, 3250-3255, 2008.

[8] M. Yamakita: What is UKF (Unscented Kalman Filter)?, Systems, Control and Information of ISCIE, 50-7, 261-266 (in Japanese), 2006.

[9] Y. Sawada and T. Watanabe: LQG Control of a Parallel-structured Single-link Flexible Arm, Proc. 51th Annual Conference of ISCIE, 372-373, 2007.

[10] S. J. Julier, J. K. Uhlmann and H.F. DurrantWhyte: A New Approach for Filtering Nonlinear Systems, Proc. of the American Control Conference, 1628-1632, 1995.

[11] S. J. Julier, J. K. Uhlmann and H. F. DurrantWhyte: A New Extension of the Kalman Filter to Nonlinear Systems, Proc. AeroSense: 11th Int. Symp. Aerosp./Defense Sensing, Simulat. Contr., Orlando, FL, 1997.

[12] S. J. Julier, J. K. Uhlmann and H. F. DurrantWhyte: A New Method for the Nonlinear Transformation of Means and Covariances in Filters and Estimators, IEEE Trans. on Automatic Control, 45-3, 477-482, 2000.

[13] K. Nonami and H. Tian: Sliding Mode Control: Nonlinear Robust Control Theory, Corona Pub., (in Japanese), 1994.

[14] Y. Saad, Iterative Methods for Sparse Linear Systems, PWS Publishing Company, 1996. 